\title{
E-TENDERING USING BLOCKCHAIN
}

\author{
SNEHAL BHOGAN ${ }^{1}$, ASHISH NARVEKAR ${ }^{1}$, YUVRAJ ARONDEKAR ${ }^{2}$, TEJAS KHEDEKAR ${ }^{2}$, \\ SURVISHIRODKAR ${ }^{2}$, NIKITA MAYEKAR ${ }^{2} \&$ DAMODAR NAIK ${ }^{2}$ \\ ${ }^{\text {I}}$ Professor, Department of Computer Engineering, AITD, Goa University, Taleigao, Goa, India \\ ${ }^{2}$ Student, Department of Computer Engineering, AITD, Goa University, Taleigao, Goa, India
}

\begin{abstract}
The process of tendering, even if it is paper based, it raises several legal issues. The main concept of tendering is to befair and transparent and requires trust between both the tenderer and bidder.

The use of electronic medium in tendering raises the need for not onlylegal issues but also the security threats that come up when moving to an open network environment.

A block chain is a growing list of record, called blocks, which are linked using cryptography. Block chain techniques maintain transparency of data on all its peer on the network.

In the current system of e-Tendering: The tendering organization with developer level access can see the tender whenever they want, they can read bids before the deadline of tendering process, and it takes lot of time to complete the procurement action.

In our project using block chain we are making the e-Tendering process secure in the following manner. Tendering organization cannot change the tender once it is based on block chain, it cannot read the bid until a deadline is expired, and the bidders cannot see or change the bids of other organizations.

KEYWORDS: Blockchain, Tender \& Bid
\end{abstract}

Received: Apr 19, 2019; Accepted: May 10, 2019; Published: Jun 14, 2019; Paper Id.: IJCSEITRDEC20193

\section{INTRODUCTION}

Tendering is a process in which bids are accepted from different organizations to perform a specific work or to provide the specific services. Tendering process consist of tendering organization and bidding organization. Tendering organization keeps the hold of all the process. Tendering organization places a document known as tender. Tender specifies the services and product requirements required to place the Bid. Once the tender has placed the bidding organization places the bid for desired tender.

We are using blockchain technology to store the data securely. Blockchain is a growing list of records, called blocks which are interconnected using cryptographic hash. In blockchain there is no centralized ledger instead information is stored on decentralized ledger. It means that same data is shared between all the members of the decentralized network. In blockchain there is no central body overseeing the result. There is one chain specified for organisation. It is the most secured way. If once you place a tender you cannot update it. If there is some emergency then they have to place a new tender. The tendering organisation cannot see the bids till deadline. After the deadline no bids are accepted. Bidders cannot see or read the bids of other organisation, also they cannot see who else have placed a bid. 


\section{PROJECT FLOW}

\section{Flow of System}

The organization who wants to publish a tender is a tendering organization and the organization who wants to bid for tender is a bidding organization.

\section{Tendering Organization}

Tendering organization will prepare a bid to be published so that bidders can bid. They will first register themselves on website or if they have already registered then they will login. Now they can upload their tender on the website. All information about tender will be taken, like opening date of tender, closing date of tender etc.

\section{Bidding Organization}

On opening date the tender will be opened and any bidder who has registered can bid for it. For that, biddingorganization has to register first thenonlytheycanbid for the tender. All the information about bidder will be stored in the database.

The bidder first upload the document, at that time the document will be converted to a hash value in the background of the website and this hash value will be send to database and stored accordingly.

\section{After Deadline}

After the deadline, bidding organization has to again send the same document for verification. After deadline, tendering organization will have the hash of previously uploaded document and now it will have the actual document so now tendering organization can calculate the hash of newly uploaded document and verify against the database to check if there are any changes in the document.
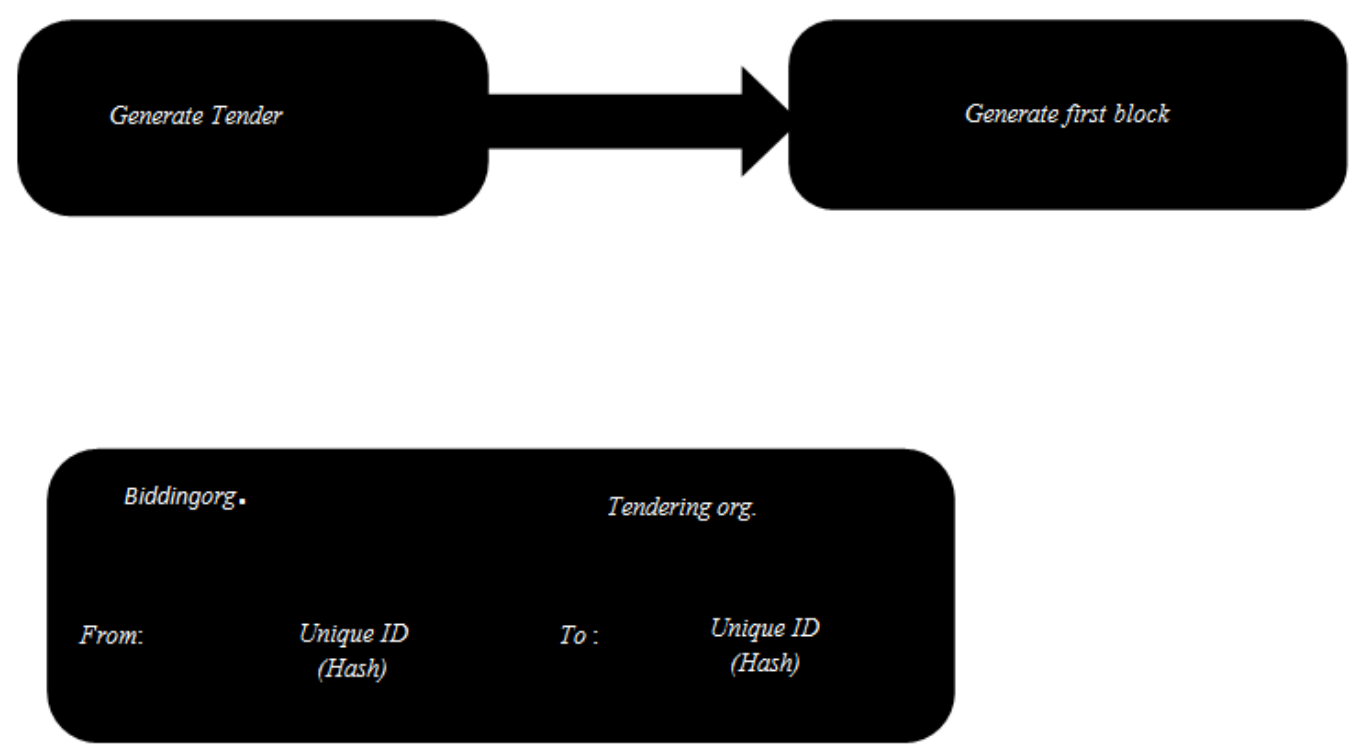

Figure 1: Tendering Organization 


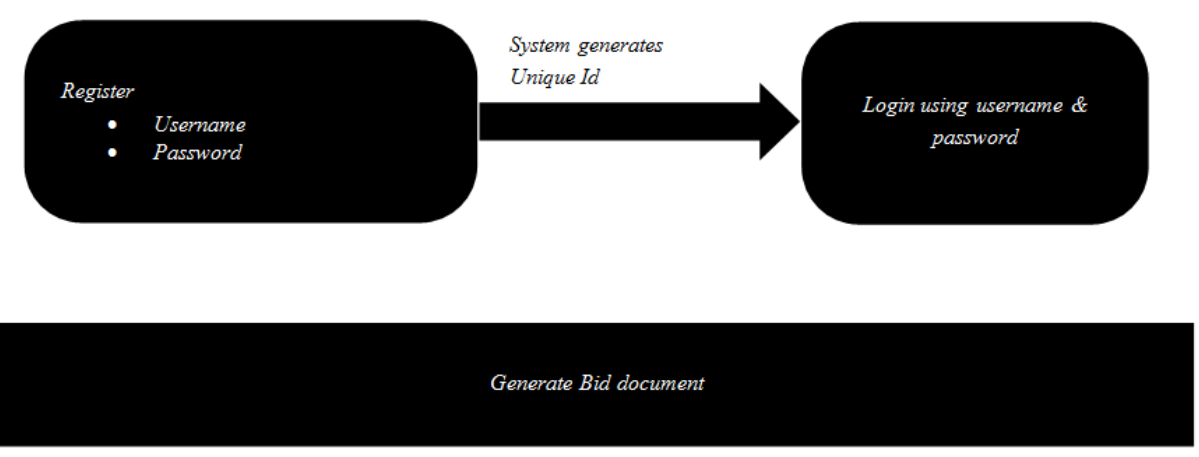

Figure 2: Bidding Organization

Ask all Bidding organizations to send plain text document

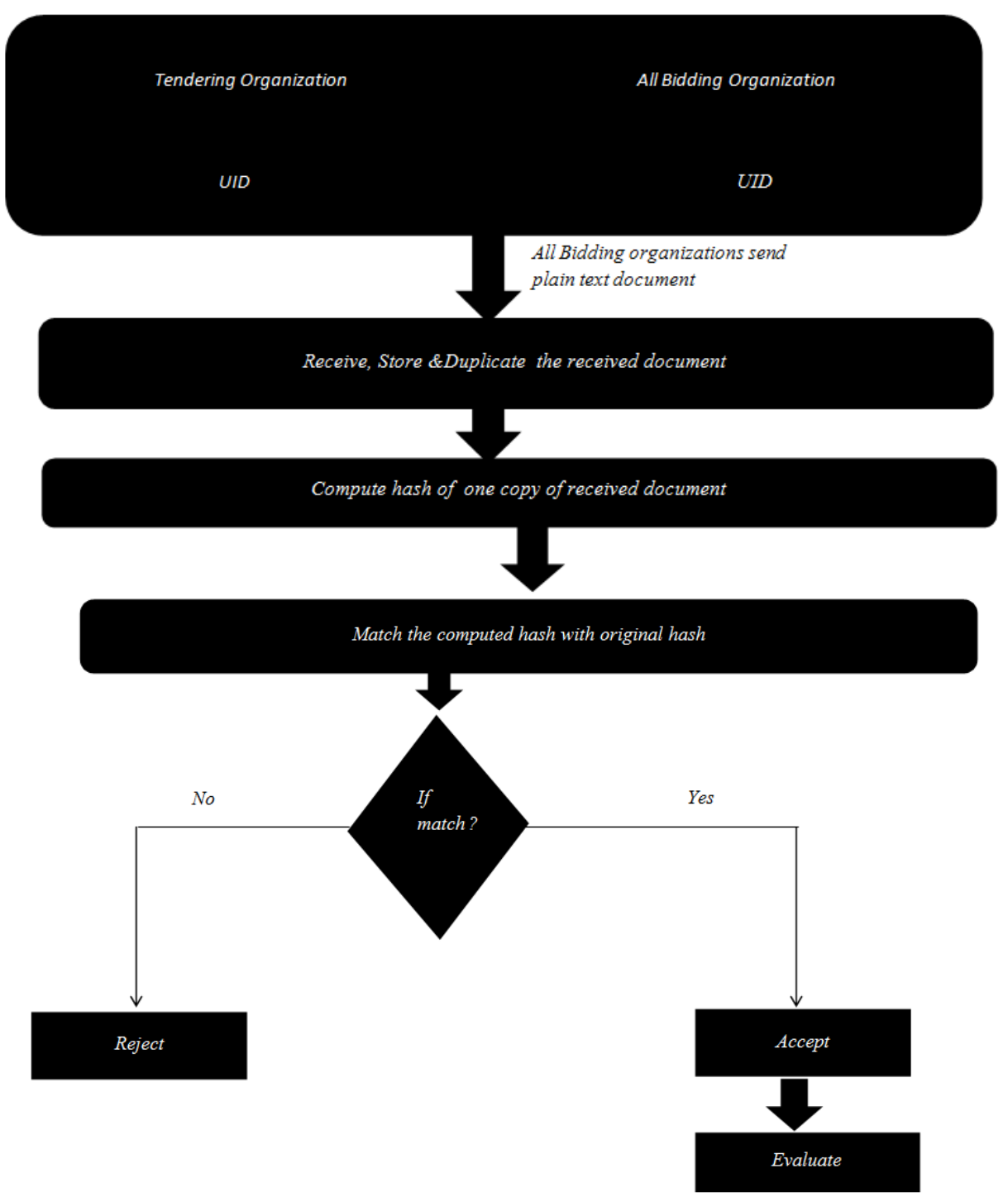

Figure 3: Process Flow 


\section{CONCLUSIONS}

E-tendering using blockchain over comes the issues with older tendering systems. It increases the efficiency in terms of security of data and storage. In older systems chances of misuse of data was high, which is eliminated using blockchain in our system. In future, the system can be modified using smart contracts. Smart contacts will help to automatically compute the results eliminating illegal activities completely.

\section{REFERENCES}

1. Definingsecurity services for electronictenderingRong Du, Ernest Foo, Colin Boyd, Brian Fitzgerald, Information securityResearch Centre(ISRC), Faculty of Information Technology, Faculty of law, Queensland University of Technology.

2. Fair and Transparent BlockchainbasedTendering Framework - A StepTowards Open Governance by FreyaSheerHardwick, Raja NaeemAkram, and Konstantinos Markantonakis, ISG-SCC, Royal Holloway, University of London, Egham, United Kingdom

3. Multichain : https://www.multichain.com/developers/ 